\title{
APPLICATION OF PROBABILISTIC ANALYSIS TO AIRCRAFT IMPACT DYNAMICS
}

\author{
Karen H. Lyle a \\ Sharon L. Padula ${ }^{b}$ \\ NASA Langley Research Center, Hampton VA, 23681 \\ Alan E. Stockwell ${ }^{\mathrm{c}}$ \\ Lockheed Martin Space Operations, Hampton VA, 23681
}

\begin{abstract}
Full-scale aircraft crash simulations performed with nonlinear, transient dynamic, finite element codes can incorporate structural complexities such as: geometrically accurate models; human occupant models; and advanced material models to include nonlinear stressstrain behaviors, laminated composites, and material failure. Validation of these crash simulations is difficult due to a lack of sufficient information to adequately determine the uncertainty in the experimental data and the appropriateness of modeling assumptions. This paper evaluates probabilistic approaches to quantify the uncertainty in the simulated responses. Several criteria are used to determine that a response surface method is the most appropriate probabilistic approach. The work is extended to compare optimization results with and without probabilistic constraints.
\end{abstract}

\section{Introduction}

One goal of the NASA Aviation Safety Program is the development of a validated crash simulation methodology, Ref. [1]. The validated simulation methodology can be utilized to aid in the future design and certification process, by reduced design and certification cycle times and decreased costs. In order to validate the modeling approaches, correlation of the simulation results with experimental data is necessary. References [2] and [3] contain additional information about the correlation effort. However, a major deficiency has been identified in the current correlation approaches relating to the lack of sufficient information to adequately determine the uncertainty in the

\footnotetext{
${ }^{a}$ Aerospace engineer, Structural Dynamics Branch, MS495

${ }^{b}$ Senior research scientist, Multidisciplinary Optimization Branch, MS 159, Assoicate Fellow, AIAA

${ }^{\mathrm{C}}$ Senior engineer, Structures and Dynamics Section, MS 389, Member, AIAA
}

experimental data. Destruction of the test article during the test, in addition to the expense in performing an impact test on a full-scale fuselage or fuselage section severely limits the capability to perform repeatability testing. Moreover, to compute impact responses of complex structures requires that assumptions and simplifications be made in the model representation. Factors affecting experimental and modeling uncertainty include: off-nominal impact conditions (e.g., attitude, velocity); material property variations (e.g., yield, hardening modulus, failure, rate dependencies); and fabrication anomalies (e.g., non-uniform cross-sections, imperfect structure assembly).

Many papers concerning probabilistic analysis for aerospace or vehicular applications exist in the literature. Sample applications can be found in Refs. [4-6]. An example specifically related to crashworthiness is found Ref. [7] for an automotive application. Although extensive work has been done to enable the use of probabilistic analysis, few applications involving impact dynamics for aircraft structures exist.

The objective of the present work is to learn about probabilistic analysis and its application to impact dynamics. The plan is to begin with a simplified finite element model with 12 candidate random variables. The probabilistic analysis of this model is studied and the number of random variables is reduced. Using a reduced set of random variables, more extensive uncertainty quantification and optimization studies are possible. In the future, a high fidelity finite element model can be studied using the best methods identified in the present research.

The first section of this paper describes the finite element model and simulation details. The second section identifies the most important random variables for probabilistic analysis. The third section evaluates probabilistic approaches to approximate the bounds in responses produced by experimental and modeling 
uncertainty. The fourth section compares optimization results with and without probabilistic constraints. Reliable probabilistic analysis and optimization processes can aid in the design of future aircraft for improved crashworthiness.

\section{Description of Finite Element Simulations}

A photograph of the structure to be studied is shown in Figure 1. This is a Fokker F28 section from a recently retired aircraft. The section is primarily fabricated from aluminum and is 1.5 meters long and 3.3 meters in diameter. The finite element model and simulations were developed based on three requirements to make it suitable for this study. The finite element model and simulations must be computationally efficient, stable, and capable of capturing the basic physics of a typical test. Computational efficiency is necessary to enable completion of numerous computations. This required a Monte Carlo approach to establish the baseline for comparison with other techniques. The simulation must be stable over the span of input variables to avoid non-physical or non-feasible responses. Finally, the model must capture the basic physics of an aluminum fuselage section that is dropped vertically. Based on the above criteria, a simplified 2-D beam model of the section was selected to evaluate the probabilistic approaches, see Figure 2. Although not capable of capturing the detailed responses, the 2-D model is sufficient to understand the capabilities and limitations of using probabilistic approaches for full-scale impact dynamic applications.

The finite element model contains 166 elements and requires approximately 30 seconds per simulation. Concentrated masses are used to model the seat and occupant. Each concentrated mass represents one-half of the combined mass of the seat and occupant. The beams were defined by cross-sectional area and moment of inertia.

The approximation of the combined complex beam with the fuselage skin using only areas and moments of inertia introduced modeling uncertainty. Additional modeling uncertainty was incorporated in the material model through the yield stress. Experience has shown that off-nominal roll and pitch values at impact may affect the response. Because the model is $2-D$, only the roll could be varied. For the probabilistic analysis, the nominal impact parameters were vertical velocity of $7.6 \mathrm{~m} / \mathrm{s}$ with no roll, pitch, or yaw. The impact velocity of 7.6 $\mathrm{m} / \mathrm{s}$ equates to a drop height of nearly 3 meters. This impact velocity is considered to be severe but in the survivable range for such an aircraft. The output upon which the results were evaluated is the maximum vertical acceleration at the location indicated in Figure 2. In this paper, the symbol, $P_{a}$, stands for the probability that this acceleration is less than $29 \mathrm{~g}$. Several other locations or quantities could have been chosen, however, vertical floor level accelerations are frequently selected for comparison with experimental data.

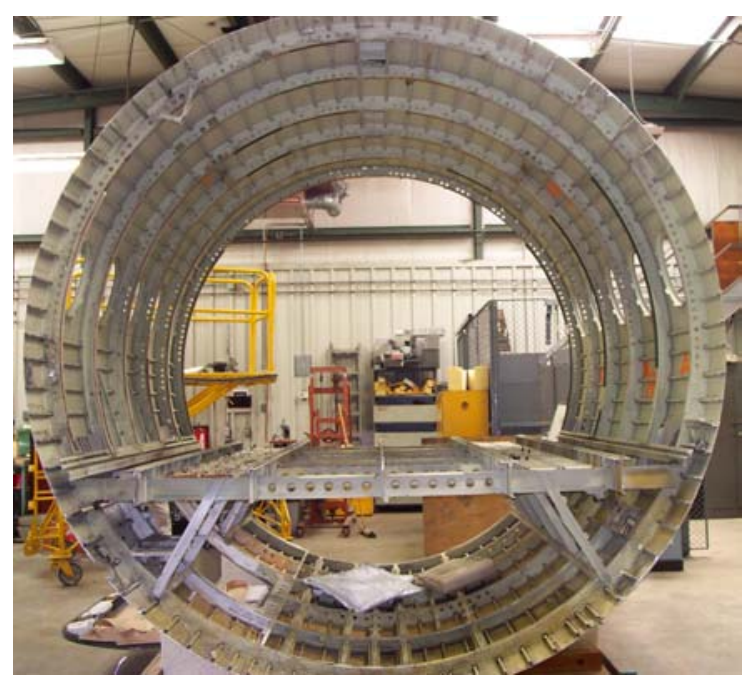

Figure 1. Photograph of Fokker F28 fuselage section.

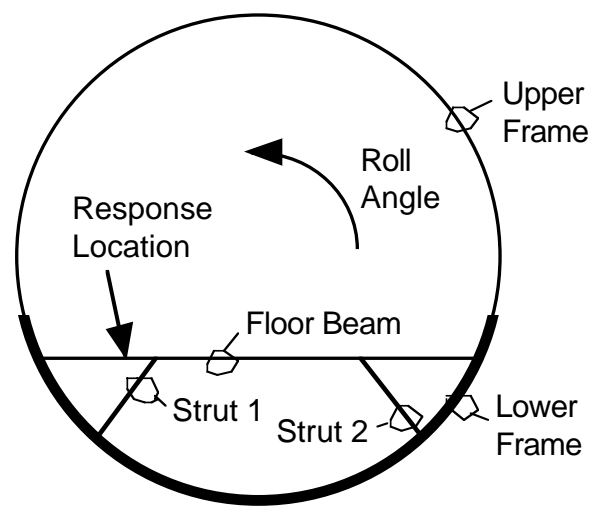

Figure 2. Fokker F28 finite element model schematic. 
The finite element simulations were conducted using MSC.Dytran*, a commercial, general purpose, non-linear transient dynamic finite element code, Ref. [8]. Some probabilistic analysis results for the experimental uncertainty computations were computed by the commercial probabilistic analysis code UNIPASS, Ref. [9]. The remainder of the uncertainty computations were performed using Matlab, Ref. [10]. The robust optimization results were computed with the commercial optimization framework iSIGHT, Ref. [11].

\section{Sensitivity Analyses for Ranking Input Variables}

An initial goal of the study was to investigate the use of sensitivity analysis results to determine the relative importance of the input variables. It was desired to significantly reduce the number of random variables used in the probabilistic analysis and thus significantly decrease the computation time. Although the relative importance is somewhat intuitive for the current finite element model and test setup, this may not be the case for future tests and more complex structures. The desire was to let the sensitivity analysis information identify the important parameters.

Table A contains a description of the 12 random variables. All variables were allowed to vary uniformly. The upper and lower bounds were established based on engineering judgment. The random variables were pseudonormalized to be bounded in magnitude between 0 and 10 . This was accomplished by using the mantissa as the random variable in the probabilistic code and ignoring the exponent. For example, random variable (RV) \#2 was allowed to vary between 2.5 and 4.5 . It was then multiplied by the factor $10^{8}$ before being input to the finite element code. Because the variables describe experimental and modeling uncertainty, the range was kept small.

\section{Baseline Sensitivity Analysis Results}

A Monte Carlo analysis was used as the baseline to evaluate the various approaches. For this particular application, 453 finite element simulations using randomly generated input

\footnotetext{
* The use of trademarks or names of manufacturers in the report is for accurate reporting and does not constitute an official endorsement, either expressed or implied, of such products or manufacturers by the NASA.
}

values provided sufficient data to accurately estimate the cumulative distribution function (CDF).

Table A. Description of 12 initial uniformly varying random variables.

\begin{tabular}{|c|c|c|c|c|}
\hline \multicolumn{2}{|c|}{ Random Variable } & \multicolumn{2}{|c|}{ Bounds } & \multirow[b]{2}{*}{ Factor } \\
\hline \# & Name & Lower, $\mathrm{b}_{\mathrm{L}}$ & Upper, $b_{U}$ & \\
\hline 1 & $\begin{array}{r}\text { Roll angle, } \\
\text { degrees }\end{array}$ & -3.00 & 3.00 & 1 \\
\hline 2 & Yield Stress, $\mathrm{Pa}$ & 2.5 & 4.5 & $10^{8}$ \\
\hline 3 & $\begin{array}{r}\text { Floor Beam } \\
\text { Area, m2 }\end{array}$ & 2.33 & 2.88 & $10^{-4}$ \\
\hline 4 & $\begin{array}{r}\text { Floor Beam } \\
\text { Inertia, } \mathrm{m}^{4}\end{array}$ & 4.27 & 5.27 & $10^{-7}$ \\
\hline 5 & Strut 1 Area, $\mathrm{m}^{2}$ & 1.60 & 1.97 & $10^{-4}$ \\
\hline 6 & Strut 1 Inertia, $\mathrm{m}^{4}$ & 6.51 & 8.03 & $10^{-8}$ \\
\hline 7 & Strut 2 Area, $\mathrm{m}^{2}$ & 1.60 & 1.97 & $10^{-4}$ \\
\hline 8 & Strut 2 Inertia, $\mathrm{m}^{4}$ & 6.51 & 8.03 & $10^{-8}$ \\
\hline 9 & $\begin{array}{r}\text { Lower Frame } \\
\text { Area, } \mathrm{m}^{2}\end{array}$ & 3.07 & 3.78 & $10^{-4}$ \\
\hline 10 & $\begin{array}{r}\text { Lower Frame } \\
\text { Inertia, } \mathrm{m}^{4}\end{array}$ & 3.38 & 4.18 & $10^{-7}$ \\
\hline 11 & $\begin{array}{r}\text { Upper Frame } \\
\text { Area, } \mathrm{m}^{2} \\
\end{array}$ & 3.88 & 4.32 & $10^{-4}$ \\
\hline 12 & $\begin{array}{r}\text { Upper Frame } \\
{\text { Inertia }, \mathrm{m}^{4}}^{4}\end{array}$ & 2.99 & 4.78 & $10^{-7}$ \\
\hline
\end{tabular}

In addition to the CDF, the correlation coefficient between the maximum acceleration and each random variable was computed from the Monte Carlo data, see Figure 3. The correlation coefficients were used to aid in the assessment of the sensitivity analyses. The correlation coefficient is the ratio of the covariance divided by the product of the standard deviation of the input variable and the output variable, Reference [12]. If a perfect linear relationship exists between the maximum acceleration and the input variable then the correlation coefficient is 1 . Because these variables were allowed to vary relatively small amounts, variation of the maximum acceleration based on any particular input is assumed to be fairly linear. Therefore deviations of the correlation coefficient from 1 are considered to result from the input variable producing little impact on the maximum acceleration. Sample scatter plots for 453 Monte Carlo data points are shown in Figures 4 and 5. The strong 
dependence of the maximum acceleration on the material yield, as seen in Figure 4, is reflected in a correlation coefficient near 1 . The roll angle (RV \#1) appears to have no effect on the maximum acceleration, as shown in Figure 5 , and is reflected in the correlation coefficient with a value near 0 .

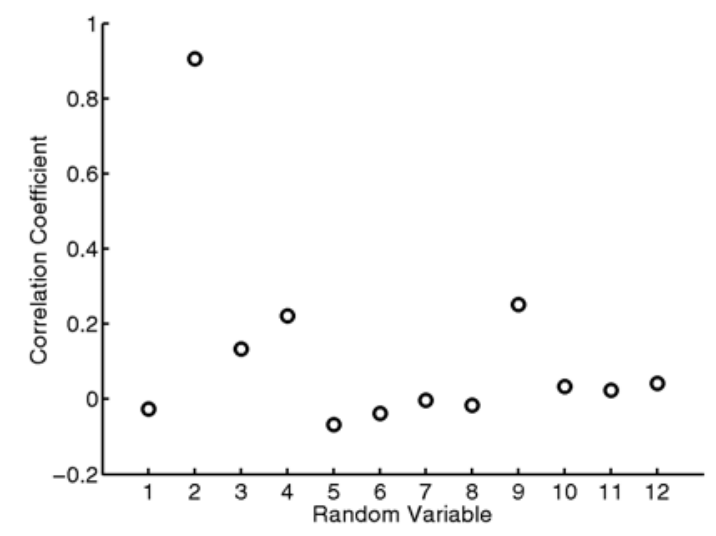

Figure 3. Correlation coefficient of maximum acceleration to random input variables (see Table A for corresponding random variable descriptions).

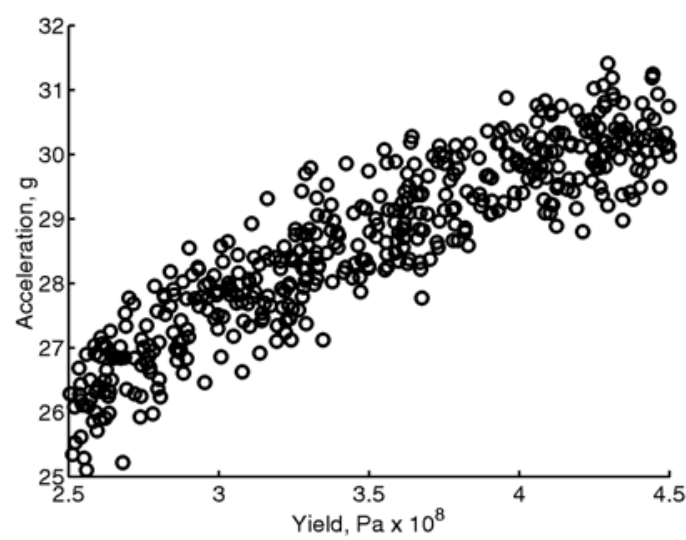

Figure 4. Scatter plot of maximum acceleration versus yield (RV \#2).

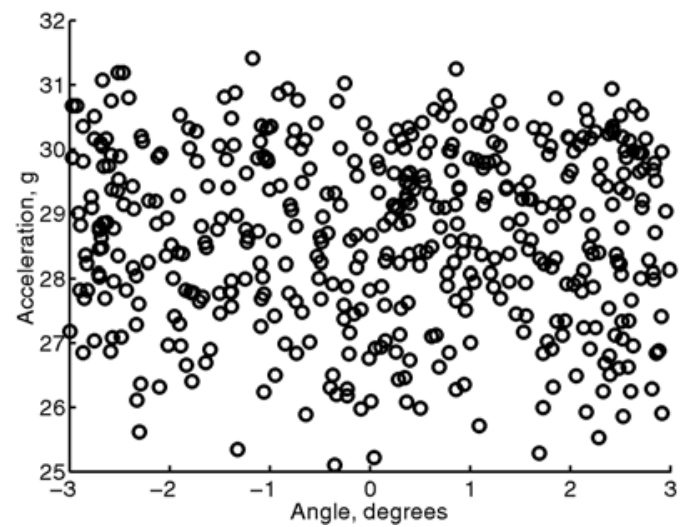

Figure 5. Scatter plot of maximum acceleration versus roll angle ( $R V \# 1)$.

\section{First-Order Reliability Method (FORM) Sensitivity Analysis Results}

The first probabilistic analysis attempted was a First Order Reliability Method (FORM) analysis in UNIPASS. FORM uses a gradientbased method to locate the most probable point (MPP) and the sensitivity information is based on the partial derivatives of acceleration with respect to each variable at the MPP. The FORM computations were performed after transformation of the uniformly distributed input variables to standard normal space. This transformation is not exact. In addition, inputoutput relationships that appear linear and simple in the original space can become complicated in standard normal space, Ref. [4]

The FORM sensitivity analysis results are suspect because the predicted probability of failure did not agree with the Monte Carlo CDF results. Thus, no sensitivity information will be presented from this approach. Five reasons for the failure of the UNIPASS implementation of the FORM analysis are postulated. 1) The transformation of the input variables to standard normal space is not exact. 2) The gradients for the input variables with negligible effect on the acceleration are contaminated by "numerical noise". 3) The dominance of one input variable on the maximum acceleration can result in a degenerate system of equations to solve. 4) The probability of failure predicted by Monte Carlo analysis is relatively large and FORM is more appropriate when the probability of failure is quite small. 5) Some combination of user inexperience and software design prevent the success of the analysis. 
Response Surface Method (RSM) Sensitivity Analysis Results

The 453 data points generated for the Monte Carlo analysis are used in the iSIGHT software to produce a quadratic response surface and to perform a FORM analysis. The results from this approach match the baseline Monte Carlo CDF results. The related sensitivity information is shown in Figure 6. The sensitivities in Figure 6 correspond to the Type 5, $\sigma(\partial g / \partial x)$, sensitivities reported by the UNIPASS software. They are found by multiplying the gradients at the MPP by the standard deviation of each random variable and then normalizing so that the maximum value equals one.

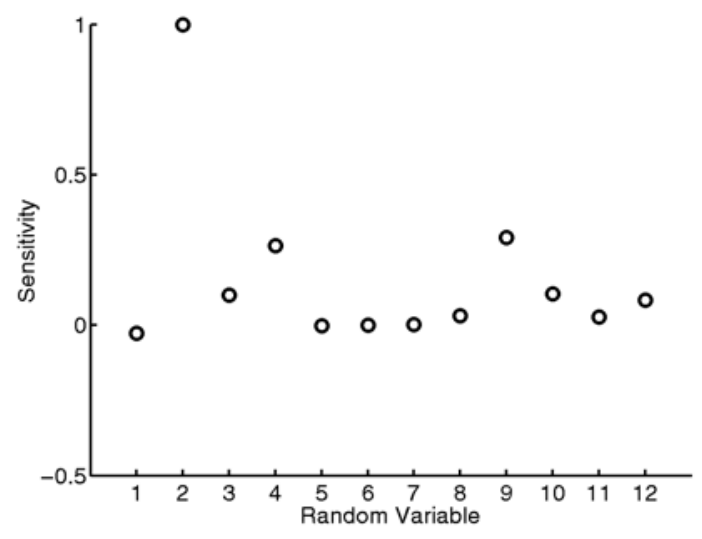

Figure 6. Sensitivity of maximum acceleration to random variables (see Table A for corresponding random variable descriptions).

The results in Figure 6 clearly indicate that RV \#2 (yield stress) is the most important variable. The plotted results correspond to the MPP for the probability that the acceleration is less than $29 \mathrm{~g}$ (this is near the $P_{a}=0.5$ or the $\leq$ $50 \%$ probability point). This plot was selected to be consistent with results in the remainder of the paper. However, plots for several different limit states were examined and all were similar to Figure 6.

In summary, the Monte Carlo probability analysis requiring 453 simulations was considered as the baseline against which the other methods were evaluated. Although the RSM sensitivity analysis based on all 453 data points was feasible for this application, it is not an approach that can be applied to typical impact problems due to the intense computational requirements.
The sensitivity results in Figure 6 were used to establish the reduced set of random variables to be used in the remaining computations. As stated earlier, a reduced set was needed for computational efficiency. The variables selected for carry-over were RV\#2 (Yield), RV \#3 (Floor Beam Area), RV \#4 (Floor Beam Inertia), and \#9 (Lower Frame Area).

\section{Maximum Acceleration Uncertainty Bounds}

The uncertainty bounds for the maximum acceleration were based on computations using the reduced set of random variables - RV \#2, \#3, \#4, and \#9 - as mentioned in the previous section. A Monte Carlo analysis based on 1000 simulations was used as the baseline. One thousand simulations were found to be sufficient, as the results for 500 simulations differed negligibly from that for 1000 .

Several probabilistic analysis methods were used to determine the best approach for this and similar applications. The evaluations of the approaches were based on several criteria. With regard to accuracy, the desired result should be a reasonable bound of the experimental and modeling uncertainty that can be used by the analyst to evaluate modeling capabilities, such as $5 \%$ and $95 \%$ bounds. The method cannot require hundreds or thousands of simulations, because of the length of time needed for a typical simulation. Although, the modeling and simulations presented in this paper have been specifically simplified to enable several approaches to be evaluated, a typical full-scale finite element simulation can require several hours to complete. Finally, many different output responses need to be bounded.

The approach that best satisfied the criteria was determined to be the Response Surface Method (RSM) approach that incorporated a Monte Carlo analysis for the probability computations (i.e., once the response surface was calculated, a Monte Carlo approach was used to compute the CDF). This method was selected because it is fairly efficient for a small number of random variables; the responses are expected to vary smoothly and relatively linearly over the span of input variables and therefore should be well approximated with a $2^{\text {nd }}$ order response surface; and unlike analyses such as FORM, the selection of input values is independent of response location, so 
that the responses for several locations can be computed for one analysis.

\section{Response Surface Method Results}

The remainder of the section will concentrate on results based on a response surface method approach. The response surface was computed using Box-Behnken to select the finite element simulation input values. Each variable was allowed to take on one of three values: mean, upper bound, or lower bound. For 4 random variables, 25 simulations were required. The response surface coefficients were computed using a $2^{\text {nd }}$-order regression to fit the data, Ref. [13]. As a check, the 1000 baseline Monte Carlo variable values were used as input to the RSM computation. The maximum accelerations computed by the finite element code were subtracted from the maximum accelerations computed by the response surface, see Figure 7 . The mean of the difference is $0.026 \mathrm{~g}$ with a standard deviation of $0.1079 \mathrm{~g}$.

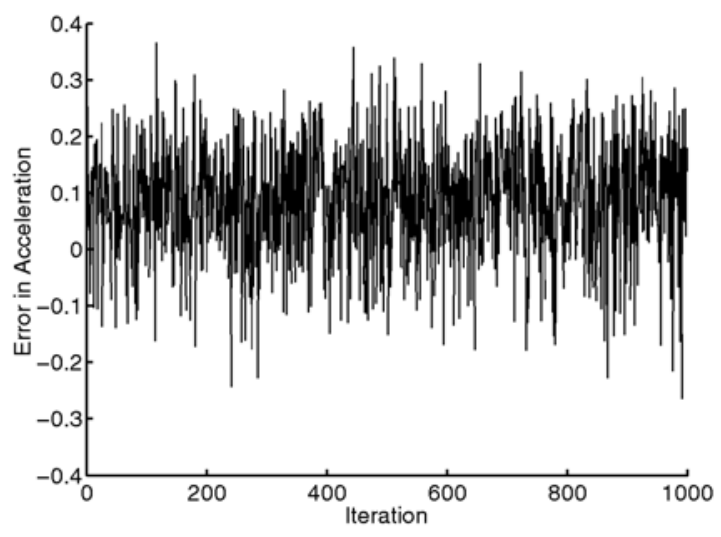

Figure 7. Difference between RSM CDF results and Monte Carlo values for uniform input variable distributions.

Once the response surface coefficients have been computed, a Monte Carlo analysis using 7500 random samples was performed to compute the maximum acceleration samples. CDF results for both the baseline Monte Carlo analysis and the RSM approach are shown in Figure 8. A summary of the results at CDF values 0.05 and 0.95 are shown in Table $B$. The difference in the bounds shown in Table $B$ between the RSM and Monte Carlo values are less than $1 \%$.
The usefulness of a chart such as Table $B$ is that it provides a range of analytical values within which the experimental data should fall. For example, the maximum acceleration should fall within the range from 26.00 to $30.34 \mathrm{~g}$. If the experimental data falls within this band, then the analytical results would be considered as accurate as can be expected based on the experimental and modeling uncertainty.

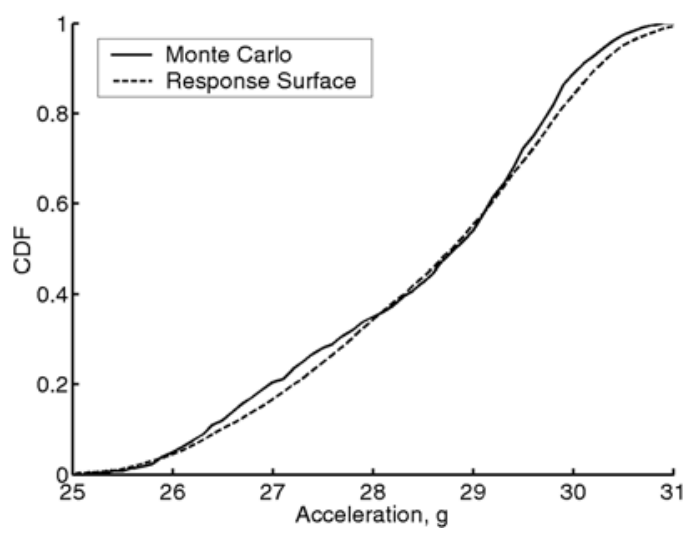

Figure 8. Comparison of Monte Carlo and RSM cumulative distribution functions.

Table B. Experimental uncertainty bounds for random variables with uniform distributions.

\begin{tabular}{|l|c|c|}
\hline \multirow{2}{*}{ Method } & \multicolumn{2}{|c|}{ Bound } \\
\cline { 2 - 3 } & $5 \%$ & $95 \%$ \\
\hline Monte Carlo & 26.00 & 30.34 \\
\hline Response Surface & 26.03 & 30.50 \\
\hline
\end{tabular}

The results presented here indicate that a Response Surface approach shows promise for quantifying the experimental and/or modeling uncertainty for impact dynamics. However, care must be exercised in selecting the number of random variables. In addition, the desired output from the simulation must vary smoothly and slowly to make the response surface accurate.

\section{Optimization Under Uncertainty}

In addition to utilizing simulations in the certification process, it has been proposed to enhance the design process by incorporating uncertainty in the optimization process. Therefore, once probabilistic measures of crashworthiness such as those presented in the previous section are available, it is interesting to consider how the resulting designs will differ 
from designs created by a more traditional approach. One possible way to investigate this question is by studying optimization solutions with and without probabilistic constraints. In this section, the capabilities and limitations of optimization under uncertainty for this application are illustrated by studying two optimization problems. The approach using deterministic constraints is delineated as Problem A, while the incorporation of uncertainty in the constraints will be referred to as Problem B.

The optimization application is different from the uncertainty computations previously presented in both the number and the definitions of the input variables. The new definitions are described in Table C. For example, RV \# 3, 4 and 9 are considered design variables, $v$, and therefore have a larger variation than is considered appropriate for the modeling uncertainty. RV \#2 plus concentrated mass and impact velocity are included as random variables, $u$. These random variables incorporate factors that can affect the response but are not part of the design space. For example, concentrated mass is precisely known during a test, however, using a fixed value for the passenger plus seat mass may not be justified during the design process. Although the design variables were specified as having a uniform distribution, the probabilistic code transforms the variables to a normal distribution where the standard deviation $(\sigma)$ is compute as follows: $\sigma=\left(b_{\mathrm{u}}-b_{\mathrm{L}}\right) /(2 \sqrt{3})$.

\section{Optimization Formulations}

The deterministic optimization Problem

A, is:

$$
\begin{array}{ll}
\text { minimize } & W \\
\text { subject to } & a \leq a_{\text {allowable }} \\
& d \leq d_{\text {allowable }} \\
\text { for } & v_{\min } \leq v_{i} \leq v_{\max } \\
& u_{j} \leq u_{\text {mean }}
\end{array}
$$

where $W$ is the weight of the structure (not including concentrated mass), $a$ is the acceleration at the passenger seat location, $d$ is the displacement of the subfloor at the monitoring station, and $v_{i}$ are the normalized cross-sectional areas and inertias that are used as design variables. The $a_{\text {allowable }}$ is acceleration that is considered survivable and $d_{\text {allowable }}$ is the maximum displacement. For example, if the clearance between the subfloor and the fuselage

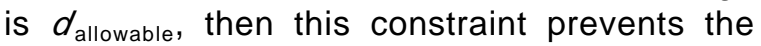

\begin{tabular}{|c|c|c|c|c|}
\hline \multirow{2}{*}{$\begin{array}{c}\mathrm{I} / \mathrm{O} \\
\text { symbol }\end{array}$} & \multirow[b]{2}{*}{ Definition } & \multirow[b]{2}{*}{ Mean } & \multicolumn{2}{|c|}{ Bounds } \\
\hline & & & Lower, $b_{L}$ & Upper, $b_{u}$ \\
\hline$v_{1}$ & $\begin{array}{l}\text { Floor Beam } \\
\text { Area, } \mathrm{m}^{2}\end{array}$ & $3.00 e-4$ & $2.42 e-4$ & $3.58 e-4$ \\
\hline$v_{2}$ & $\begin{array}{l}\text { Floor Beam } \\
\text { Inertia, } \mathrm{m}^{4} \\
\end{array}$ & $4.77 e-7$ & $4.48 \mathrm{e}-7$ & $5.06 e-8$ \\
\hline$v_{3}$ & $\begin{array}{c}\text { Lower Frame } \\
\text { Area, } \mathrm{m}^{2}\end{array}$ & $3.50 e-4$ & $2.92 \mathrm{e}-4$ & $4.08 \mathrm{e}-4$ \\
\hline$\overline{u_{1}}$ & $\begin{array}{c}\text { Yield } \\
\text { Strength, } \mathrm{Pa}\end{array}$ & $3.50 \mathrm{e} 8$ & $2.92 \mathrm{e} 8$ & $4.08 \mathrm{e} 8$ \\
\hline$u_{2}$ & $\begin{array}{c}\text { Impact } \\
\text { Velocity, } \mathrm{m} / \mathrm{s}\end{array}$ & -7.00 & -8.15 & -5.85 \\
\hline$u_{3}$ & $\begin{array}{l}\text { Concentrated } \\
\text { Mass, kg }\end{array}$ & 50.0 & 35.6 & 64.4 \\
\hline$W$ & $\begin{array}{l}\text { Structural } \\
\text { Weight, N }\end{array}$ & 136 & 125 & 148 \\
\hline$a$ & Acceleration, $\mathrm{g}$ & 28.1 & 20.2 & 39.1 \\
\hline$d$ & $\begin{array}{c}\text { Displacement, } \\
\text { m }\end{array}$ & -.228 & -.323 & -.137 \\
\hline$a_{\text {allowable }}$ & & 29.0 & & \\
\hline$d_{\text {allowable }}$ & & -0.24 & & \\
\hline
\end{tabular}
optimization routine from calculating unrealistic displacements.

Table C. Summary of input and output to MSC.Dytran software.

In optimization Problem $\mathbf{A}$, the design variables, $v$, correspond to RV \# 3, 4 and 9 that proved to have a significant effect on the acceleration. The factors, $u_{i}$, are inputs that are assigned fixed values based on estimates of their mean values. For example, the concentrated masses that replace the seats and the passengers are fixed at $50 \mathrm{~kg}$.

The probabilistic optimization Problem

$B$, is:

minimize $\quad W$

subject to $\quad a \leq a_{\text {allowable }}$

$P_{d} \geq 75 \%$

for

$$
\begin{aligned}
& v_{\min } \leq v_{i} \leq v_{\max } \\
& u_{j} \in N\left[u_{\text {mean }}, u_{\sigma}\right]
\end{aligned}
$$

where the uncontrollable factors $u$ are described by a normal distribution with mean $u_{\text {mean }}$ and standard distribution $u_{\sigma}$ and the constraint on displacement is replaced with a probabilistic 
constraint. Here $P_{d}$ is the probability that displacement constraint is satisfied. Thus, the probabilistic constraint in Problem $\mathbf{B}$ has the same effect as the constraint in Problem $\mathbf{A}$ but it requires a $75 \%$ probability of satisfaction.

Problem B will provide a more conservative design that takes into account variations in yield stress, impact velocity and passenger mass. It is likely that the probabilistic constraint will result in a higher structural weight design but it will also provide a physically meaningful and more robust design.

Optimization problems $\mathbf{A}$ and $\mathbf{B}$ will be solved using a formulation recommended by Eldred, Ref. [14]. As for the uncertainty analysis, this formulation (see flow chart in Figure 9) uses an approximate model to replace repeated simulations using the finite element code thus reducing computational expense, mitigating the effects of nonsmooth response variations and facilitating experimentation with optimization options. The arrows indicate the flow of data between these modules. This formulation was implemented using the iSIGHT commercial software package developed by Engineous Software, Inc. [11]. The iSIGHT software implements a "single-loop-singlevector" approach based on Ref. [15] where the optimization algorithm is the Modified Method of Feasible Directions, uncertainty quantification is either FORM or a Monte Carlo analysis, and the approximate solution is a Kriging model.

The approximate models are based on 49 simulations of the finite element code as summarized in Table $\mathrm{C}$. The uniformly distributed input variables were transformed for the uncertainty analysis to a normal distribution for each $u$ with a mean given in Table $D$ and a standard deviation based on the minimum and maximum values listed.

This set of 49 inputs and outputs includes many designs that satisfy the constraints in optimization Problem A. Of the acceptable designs, one with a small weight is used as an initial guess for all optimization trials (see Table D). The design variables in Table D are normalized so that they vary between 0 and 1.

\section{Optimization Results}

Figure 10 shows convergence histories for the structural weight, displacement and acceleration for both optimization Problems A and $\mathbf{B}$. The square symbols indicate outputs from the deterministic problem and the circles indicate outputs from the probabilistic problem. As expected, Problem A converges to a solution with less structural weight and with a displacement that is exactly equal to $d_{\text {allowable. }}$. Problem B converges to a solution with increased weight but with a greater probability that the calculated displacement is physically possible. The results are also summarized in Tables $D$ and $E$. In each case, approximate values of the optimized weight and final values of $a$ and $d$ are confirmed by additional executions of the finite element code. Moreover, the probability of success measures, $P_{a}$ and $P_{d}$, are estimated using Monte Carlo analysis in the iSIGHT package.

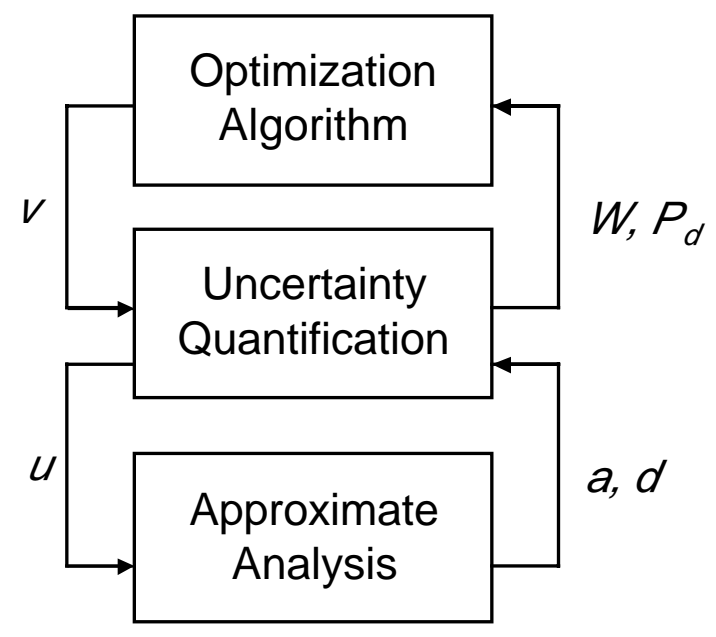

Fig. 9. Flow chart of nondeterministic optimization formulation.

Table D. Summary of optimization results.

\begin{tabular}{|c|c|c|c|c|c|}
\hline & $W$ & $v_{\text {normalized }}$ & $v_{\text {original }}$ & $P_{\mathrm{a}}$ & $P_{\mathrm{d}}$ \\
\hline Initial & 130.1 & $(0.1$, & $(2.54 \mathrm{e}-4$, & .705 & .572 \\
guess & & 0.2, & $4.60 \mathrm{e}-7$, & & \\
& & $0.3)$ & $3.27 \mathrm{e}-4)$ & & \\
\hline OPT & 125.6 & $(0.0$, & $(2.42 \mathrm{e}-4$, & .771 & .514 \\
(A) & & 0.273, & $4.64 \mathrm{e}-7$, & & \\
& & $0.0)$ & $2.92 \mathrm{e}-4)$ & & \\
\hline OPT & 142.1 & $(0.0$, & $(2.42 \mathrm{e}-4$, & .513 & .767 \\
(B) & & 0.0, & $4.488 \mathrm{e}-7$, & & \\
& & $1.4)$ & $4.54 \mathrm{e}-4)$ & & \\
\hline
\end{tabular}




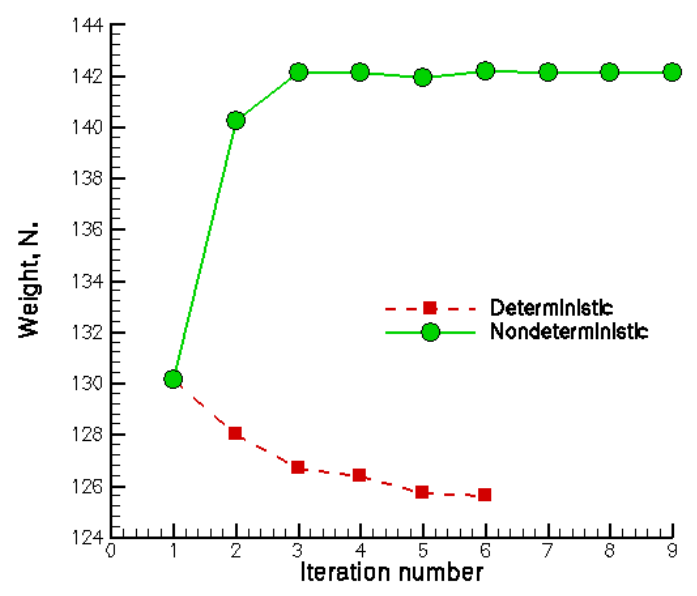

10a. Structural weight as a function of optimization iteration.

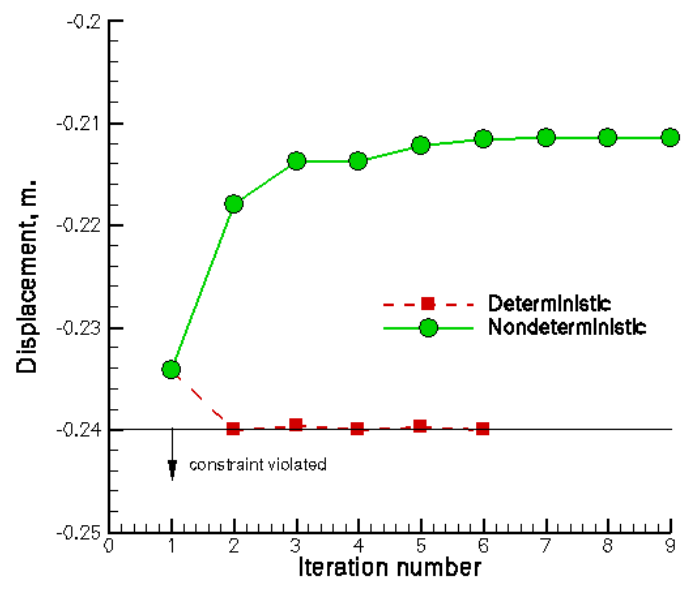

10b. Displacement constraint as a function of optimization iteration.

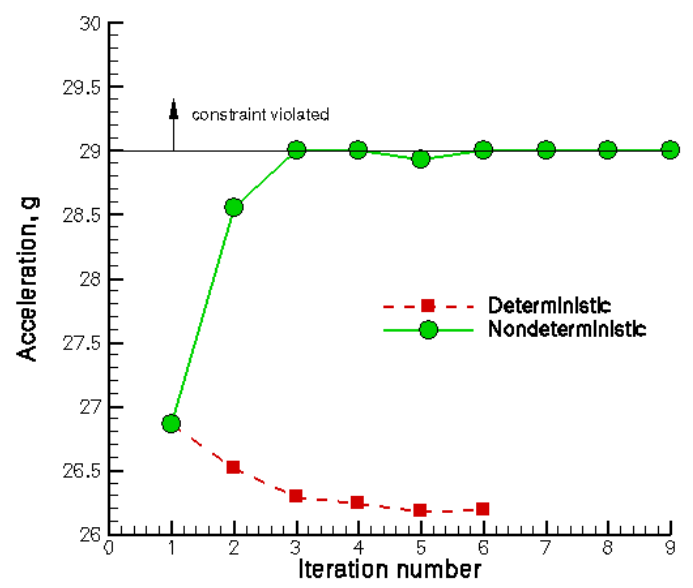

10c. Acceleration constraint as a function of optimization iteration.

Fig. 10. Comparison of convergence histories for deterministic and probabilistic optimization.
These tests reveal that both Problem A and $\mathbf{B}$ have potential pitfalls. Problem A produces a design with low structural weight but this design is adequate in only $50 \%$ of the crash scenarios. Whenever, passenger weight or impact velocity are higher than the mean values, then the subfloor probably impacts the fuselage and the acceleration calculated by the finite element is invalid. Problem B produces a much more reliable design, but the problem is computationally expensive and requires more iterations of the optimization process. Figure 11 sheds some light on both of these observations. Figure 11a is a 3-D contour plot of displacement as a function of the three normalized design variables and $11 \mathrm{~b}$ is a similar contour plot of $P_{\alpha^{*}}$ First, notice that all the values of displacement in $11 \mathrm{a}$ are less than $d_{\text {allowable }}$ and thus all the designs are feasible for Problem A. Next, notice that none of the values of $P_{d}$ in $11 \mathrm{~b}$ exceed $75 \%$ and thus none of these designs is feasible for Problem B. Finally, notice that $11 \mathrm{~b}$ is more nonlinear than 11a. These observations explain why Problem B converges more slowly and why it converges to a solution point which is outside of the range of values in Table D. In general, probabilistic optimization problems are harder to solve.

The use of approximate analysis in both optimization problems was successful. Table E indicates that the approximate analysis and the MSC.Dytran analysis match very well at both final design points. It is important to note, however, that both designs fall close to the range of values used to build the approximations. If the optimization process had converged well outside of this range then at least one more set of 49 finite element simulations and another optimization cycle would have been necessary. This process can be computationally expensive, especially if the number of design variables and random variables is large. The best strategy is to perform a deterministic optimization first and then use those results to pick an initial guess and to set the range of values used to build the approximation. 


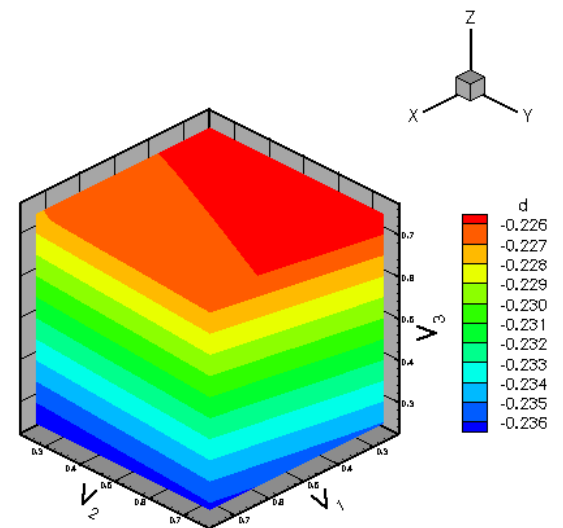

11a. Displacement as a function of design variables.

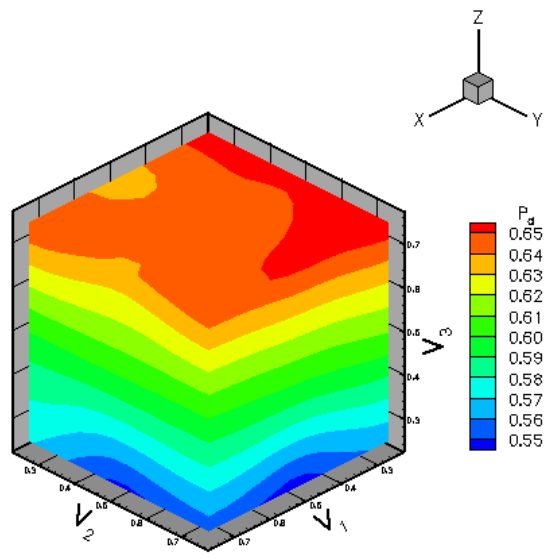

11b. Probability that displacement is less than its allowable value.

Fig. 11. Three dimensional contour plots of displacement constraint.

Table E. Comparison of Optimization response with MSC.Dytran computations.

\begin{tabular}{|l|l|l|}
\hline & $a$ & $d$ \\
\hline Initial guess & 26.87 & -0.234 \\
\hline OPT (A) & 26.20 & -0.240 \\
\hline MSC.Dytran (A) & 26.37 & -0.239 \\
\hline OPT (B) & 29.00 & -0.211 \\
\hline MSC.Dytran (B) & 28.31 & -0.215 \\
\hline
\end{tabular}

\section{Concluding Remarks}

An application of probabilistic analysis to compute the effect of experimental and modeling uncertainty on the response and for robust design optimization has been presented. The sample structure was an aluminum commuter aircraft section undergoing a severe but survivable vertical impact. The structure was modeled using an equivalent beam representation in a commercial nonlinear transient dynamic code. The output studied in this application was the vertical acceleration at the floor level. In general, the computational cost of probabilistic analysis may be too high for routine use in impact dynamics. However, the computational cost is justified when it improves the safety of the product or it reduces the number of expensive dynamic tests. Results showed that:

1) The use of sensitivity results to reduce the number of random variables is not considered feasible for this application because of the computational cost required to obtain reasonable accuracy. At the present, it seems that engineering judgment is the best way to select the input variables for this application.

2) Using a response surface approximation follows by a Monte Carlo probability computation provided the accuracy required while at the same time limiting the number of finite element simulations.

3) The addition of nondeterministic constraints to the optimization problem insures a solution that is less sensitive to uncontrollable parameters such as the weight of a passenger.

4) As was demonstrated, the nondeterministic optimization problem is harder to solve than the deterministic one because the probabilistic constraints tend to be more nonlinear than the original constraints.

5) The computational cost of probabilistic optimization increases with the number of random variables. For the results in the paper, the optimization was computed based on a response surface approximation. When the optimization was performed using a deterministic approach then only three variables were used. For three variables 13 finite element simulations are required. With the introduction of probabilistic constraints, the number of variables increased to six and required that 49 finite element simulations be performed. 


\section{$\underline{\text { References }}$}

1. Jones, L. E.: Overview of NASA Aviation Safety Program Crashworthiness Element. Proceedings of American Helicopter Society $58^{\text {th }}$ Annual Forum, Montreal Canada, June 11-13, 2002.

2. Lyle, K. H.; Bark, L. W.: Data Reduction and Its Impact on Test-Analysis Correlation. Proceedings of the FAA Fire and Cabin Safety Conference, Atlantic City, NJ, October 22-25, 2001.

3. Lyle, K. H.; Bark, L. W.; and Jackson, K. E.: Evaluation of Test/Analysis Correlation Methods for Crash Applications. Journal of the American Helicopter Society. Vol. 41 No. 4, October 2002,pp. 233-242.

4. Thacker, B. H., et al: Errors and Uncertainties in Probabilistic Engineering Analysis, Proceedings of the $42^{\text {nd }}$ AIAA/ASME/ASCE/AHS/ASC Structures, Structural Dynamics, and Materials Conference and Exhibit, NonDeterministic Approaches Forum, AIAA Paper 2001-1239, Seattle, WA, April 1619, 2001.

5. Hoser, S. et at. Observations on CFD Simulation Uncertainties. Proceedings of the $9^{\text {th }}$ AIAA/ISSMO Symposium on Multidisciplinary Analysis and Optimization, AIAA Paper 2002-5531, Atlanta GA, September 4-6, 2002.

6. Long, M. W.; and Narciso, J. D.: Probabilistic Design Methodology for Composite Aircraft Structures. DOT/FAA Report No. AR-99/2, June 1999.

7. Yang, R. J, et al. Reliability-Based Multidisciplinary Design Optimization of a Full Vehicle System. Proceedings of $43^{\text {rd }}$ AIAA/ASME/ASCE/AHS/ASC Structures, Structural Dynamics, and Materials Conference and Exhibit, AIAA Paper 2002-1758, Denver CO, April 2225, 2002.

8. Anon., MSC.Dytran User's Manual, Version 2002, Santa Ana CA, November 2001.

9. Lin, H-Z. et at: Development of UNIPASS- A Unified Probabilistic Assessment Software System. Proceedings of $42^{\text {nd }}$ AIAA/ASME/ASCE/AHS/ASC
Structures, Structural Dynamics, and Materials Conference, AIAA Paper 2001-1644, Seattle WA, April 16-19, 2001.

10. Anon: Using Matlab, Version 6, The MathWorks, Inc. Natick MA 2000.

11. Anon: iSIGHT Reference Guide, Version 7.0. Engineous Software, Inc. Morrisville, NC, September 2002.

12. Papoulis, A.: Probability, Random Variables, and Stochastic Processes. McGraw-Hill Book Company, New York, 1984.

13. Lapin, L. L.:Probability and Statistics for Modern Engineering. PWS Publishers, Boston MA, 1983.

14. Eldred, M. S.; Giunta, A. A.; Wojkiewicz, S. F.; and Trucano, T. G.: "Formulations for Surrogate-based Optimization under Uncertainty," AIAA Paper 2002-5585, $9^{\text {th }}$ AIAA/ISSMO Symposium on Multidisciplinary Analysis and Optimization, Atlanta, GA, September 46, 2002.

15. Chen, X.; Hasselman, T. K.; Neill, D. J.: "Reliability Based Structural Design Optimization for Practical Applications," AIAA Paper 97-1403, 38 ${ }^{\text {th }}$ AIAA/ASME/ASCE/AHS/ASC

Structures, Structural Dynamics and Materials Conference, Kissimmee, FL, April 1997 